\title{
Queda em Pediatria: Um desafio para a equipe multidisciplinar.
}

\author{
Renata Pereira $^{1}$
}

\section{Resumo}

Objetivo: O evento de queda é considerado atualmente um grande desafio para o aprimoramento da qualidade da assistência na área da saúde portanto este estudo foi motivado para gerar dados que suportem a melhoria na qualidade da assistência na área da saúde. Método: Levantamento de literatura referente ao tema de queda em contexto pediátrico. Os artigos foram selecionados a partir da base de dados Scielo em português e inglês. Resultados: Apesar das quedas serem a principal causa de lesões não intencionais em crianças, trabalhos publicados são escassos. Nas crianças as quedas são relacionadas claramente ao seu estágio de desenvolvimento, o nível de lesão é baixo e dentro do ambiente hospitalar a maior parte das quedas é decorrente das condições ambientais do serviço. Conclusão: Evidencia-se a importancia da detecção dos pacientes de risco por ferramentas validadas para a população pediátrica, da utilização de protocolos de prevenção de quedas e da mudança da cultura organizacional com foco na segurança do paciente.

Palavras chave: queda, pediatria, indicadores.

Pereira, Renata. "Queda em Pediatria: Um desafio para a equipe multidisciplinar", in Anais do 20.

Congresso Internacional Sabará de Especialidades Pediátricas

\footnotetext{
${ }^{1}$ Enfermeira. Bacharel em Enfermagem pela UNIFESP, Universidade Federal de São Paulo. São Paulo/ SP, Brasil. MBA em Gestão de Pessoas pela FGV, Fundação Getulio Vargas. São Paulo/ SP, Brasil.
} 


\section{Introdução}

Segurança do paciente em pediatria é uma área relativamente nova, com poucas pesquisas publicadas e pouca atenção é dada aos riscos de acidentes em hospitais que tratam exclusivamente de crianças.

Para implementar uma iniciativa de sucesso que melhore a prevenção de quedas, de forma sustentada é necessário uma cultura organizacional e práticas operacionais que promovam o trabaho em equipe e comunicação, bem como especialização individual, uma vez que a conscientização sobre sua importância é uma responsabilidade interdisciplinar.

A existência de lesões relacionadas à queda, que comprometem a segurança do paciente, é atualmente um grande desafio para o aprimoramento da qualidade da assistência na área da saúde.

Existem ferramentas de avaliação de risco para o contexto pediátrico porém ainda seria importante estudos complementares para sua validação e aprovação, contribuindo para um melhor conhecimento desta realidade e aumento da segurança do paciente.

\section{Método}

Levantamento bibliográfico referente ao tema de queda em contexto pediátrico.

\section{Resultados}

O movimento internacional de segurança do paciente, incentivado pelas empresas acreditadoras, tem estimulado muitas instituições a começar a abordar a segurança do paciente de forma abrangente e proativa incluindo programas de segurança do paciente as suas rotinas.

É imprencindível que a equipe compreenda de maneira clara as preocupações relacionadas a esse tema, pois desta forma é provável que a motivação para se envolver nos programas de segurança do paciente seja mais forte e duradoura.

As quedas são dos eventos adversos mais frequentes e relatados e estão associadas diretamente ao aumento do tempo de internação, necessidades acrescidas de cuidados de saúde, descrédito na qualidade da equipe assistencial e serviços prestados, lesão e até mesmo morte.

Esses eventos têm sido identificados como fator chave para o custo cada vez maior nos cuidados em saúde. Por essa razão, as fontes pagadoras estão exigindo das instituições hospitalares maior cuidado referente à segurança do paciente como, por exemplo, os Centros de Serviços Medicare e Medicaid (CMS), que desde 2008 possuem uma política de restrição de pagamentos por lesões decorrentes de queda, ou seja, não reembolsam os hospitais para determinados tipos de lesões traumáticas que ocorrem enquanto o paciente está no hospital.

Isso porque é considerado que os hospitais possuem a obrigação de previnir e eliminar danos e nãomaleficências, de maneira que o paciente deve ser tratado da patologia de entrada no serviço sem adquirir complicações nesse tempo de permanência no mesmo. Ainda, é importante ressaltar que os hospitais são responsáveis pela segurança de suas instalações incluindo os equipamentos e ambiente físico que devem ter mobiliário adequados para a idade dos clientes e nível de desenvolvimento dos mesmos. .

Monitorar as quedas no ambiente hospitalar como um indicador de qualidade nos permite analisar as condições do processo/ serviço e compará-las com padrões estratégicos previamente estabelecidos, contribuindo para a verificação de desvios e consequente busca de melhorias, mantendo e aprimorando o nível de qualidade do serviço prestado. Esse conceito de medir a fim de melhorar é crucial para o sucesso das organizações em saúde.

Dentre os múltiplos riscos a que os pacientes se encontram expostos no ambiente hospitalar, a queda é um evento frequente, que pode acarretar em consequências físicas, psicológicas e sociais ao paciente e sua família sendo então fundamental investir nessa área de intervenção.

Queda é definida como um evento não planejado que levou o paciente ao chão, com ou sem lesão e relacionado a fatores intrínsecos (alterações fisiológicas, alterações patológicas fatores psicológicos e efeitos colaterais de medicamentos) e/ou extrínsecos (relacionados ao comportamento e atividade do indivíduo e seu meio ambiente). 
Apesar das quedas serem a principal causa de lesões não intencionais em crianças, relatórios publicados são escassos na validação de instrumentos que avaliam esse risco na população pediátrica. A comparação dos números no contexto hospitalar está dificultada pela escassez de dados.

Nos estudos existentes a incidência é calculada utilizando número de quedas por 1000 dias de internação e os dados apontam para incidência de 0,56-1/1000. A literatura sugere igualmente que a incidência das quedas para população pediátrica é bastante menor que na população adulta porem o nível de evidência é baixo por falta de estudos publicados.

Tal como na comunidade, e o referido em literatura para as quedas em contexto hospitalar, a idade é um fator de risco estando mais elevado na população abaixo dos 3 anos sendo que nestas crianças o nível de lesão é baixo.

Seria de se esperar que a presença dos pais funcionasse como fator de proteção para as quedas, no entanto a maioria acontece na presença dos mesmos durante a realização de atividades da vida diária.

Estudos nos mostram que a incidência de eventos com lesão em crianças hospitalizada é maior que $1 \%$.

Nas crianças as quedas são relacionadas claramente ao seu estágio de desenvolvimento, principalmente relacionados às competências motoras, em especial de marcha, com as atividades que desenvolvem, a incapacidade de avaliar riscos das mesmas, curiosidade inata e níveis crescentes de independência que coincidem com comportamentos mais desafiadores.

A maioria das quedas dentro do ambiente hospitalar são decorrentes das condições ambientais, tais como leitos inadequados a idade da criança, grades destravadas, salas de jogos, determinados brinquedos e comportamento de risco dos pais que deixam esquecidos as grades laterais baixas enquanto a criança está sozinha

Os diagnósticos de doença respiratória / pulmonar e neurológico (convulsões) foram associados com um aumento na incidência de quedas em pediatria.

É importante lembrar que os visitantes/família não podem substituir os enfermeiros na prevenção de queda durante a internação hospitalar de forma eficaz, pois nada melhor para prever e evitar um evento de queda do que a avaliação clínica do enfermeiro.

Para que os gastos, lesões e danos indesejáveis aos pacientes e profissionais sejam evitados, medidas preventivas precisam ser adotadas. Para tanto se faz necessário à detecção de pacientes de risco e a adoção de procedimentos que possam eliminar ou diminuir o risco desse determinado paciente.

Ressalta-se ainda, que crianças são diferentes dos adultos e os motivos pelos quais elas caem diferem também, portanto existe a necessidade clara de uma ferramenta de risco de quedas válida e de confiança que seja capaz de prever os fatores de risco de queda em pacientes pediátricos. Essa avaliação deve ser feita no momento da admissão do paciente e repetida diariamente até a alta dele, pois suas condições clínicas podem variar durante o período de internação.

Além disso, utilizar estratégias de educação para os pacientes, famílias e enfermeiros sobre os riscos de quedas no hospital, danos relacinados, quais as intervenções são eficazes para prevenção e estratégias de redução de risco também favorece a conscientização da importancia do assunto e maior sucesso nos programas estabelecidos.

A prevenção de quedas requer uma abordagem interdisciplinar para cuidar, uma vez que algumas partes desse cuidado são altamente rotineiras mas outros aspectos devem ser adaptados para o perfil de risco específico de cada paciente. Por isso todas as equipes que, de alguma forma interagem com o paciente necessitam de treinamento específico deste programa. Desta forma também dissemina- se o conceito da importância da prevenção de queda que será incorporado na cultura do hospital.

Integrando protocolos de prevenção de queda, agrupando os pacientes com comprometimento cognitivo em chamadas zonas de segurança e fazendo avaliações pós-queda são algumas novas estratégias para reduzir o número de quedas de pacientes em hospitais. A criação de um instrumento de notificação de quedas, avaliação de suas causas e geração de informações para produção de indicadores para monitorar o desempenho representam uma oportunidade de aprendizagem para a organização, por meio da análise das informações, feedback dos resultados para os profissionais e adoção de ações de melhoria, se necessário.

A utilização das precauções de queda universal ( que se aplicam a todos os pacientes), preconizadas pela Agency for Healthcare Research and Quality em fevereiro de 2013 também se tornou uma estratégia para prevenir o risco de queda em ambientes hospitalares. 
Existem ferramentas de avaliação de risco para o contexto pediátrico, mas eles podem não ter sido tão extensivamente validadas como as escalas de Morse e Hendrich que possuem uma literatura substancial de pesquisas em adultos.

Estudos indicam que as escalas Morse e Hendrich quando aplicadas a população pediátrica não contemplam alguns fatores extrínsecos importantes para a avaliação dessa população para risco de queda, como presença de acompanhantes, atividade envolvida e equipamento envolvido. Ainda, algumas variáveis existentes não são aplicáveis a crianças pois podem ser influenciados por seu estágio de desenvolvimento, como é o caso da variável estado mental na escala de queda de Morse e confusão, desorientação e impulsividade no modelo de risco de queda de Hendrich II.

Existem em literatura publicada duas ferramentas de avaliação para risco de queda em crianças hospitalizadas sendo elas:

CHAMPS PEDIATRIC Fall Risk Assessment Tool (2006)- Avalia quatro fatores de risco: alterações no estado mental, histórico de queda, idade menor que 36 meses e deficiência de mobilidade, mas foi indicado que esta ferramenta ainda necessitaria de estudos mais aprofundados para validação.

Appendix A. CHAMPS Pediatric Fall Risk Assessment Tool

The first four items are risk factors, and the second two are nursing interventions.

\begin{tabular}{|c|c|c|c|}
\hline Risk Factor* & Yes & No & Comments \\
\hline Change in Mental Status & & & $\begin{array}{l}\text { Episodes of Disorientation, dizziness, confusion related to post-op sta- } \\
\text { tus, medication (high dose of narcotics, rapid weaning of sedation), or } \\
\text { illness. Newborn/lnfant indicators may include: irritability, agitated, } \\
\text { inconsolability, non responsive to auditory, visual or tactile stimuli. }\end{array}$ \\
\hline History of Falls & & & $\begin{array}{l}\text { Accidental Fall=a developmentally inappropriate fall. Patient has } \\
\text { experienced an accidental fall recently. }\end{array}$ \\
\hline Age less than 36 months & & & Yes-if less than 36 months of age chronologically or developmentally. \\
\hline Mobility Impairment & & & $\begin{array}{l}\text { Mobility includes ability to get in/out of bed/crib un-assisted as well } \\
\text { as ability to utilize bathroom without assistance. } \\
\text { Yes-pat needs help of furniture/walls to ambulate. } \\
\text { Yes-patient needs crutches, walker or other assistive device to ambu- } \\
\text { late. } \\
\text { Yes-pt needs assistance of one or two people to ambulate. } \\
\text { Yes-pt is less than one year of age. }\end{array}$ \\
\hline Parental Involvement & & & $\begin{array}{l}\text { - Partners in prevention, parent education. } \\
\text { Over } 80 \% \text { of children's falls in a hospital occur when a caregiver is } \\
\text { in attendance. Falls are more likely to occur because this is a new } \\
\text { place or unfamiliar. Most falls occur with getting out of bed or } \\
\text { going to the bathroom. } \\
\text { using the call light for bathroom and or getting out of bed assis- } \\
\text { tance. }\end{array}$ \\
\hline Safety & & & $\begin{array}{l}\text { Implementation of interventions: } \\
\text { - Re-educate ask for assistance in getting out of bed } \\
\text { vised. } \\
\text { - Re-assess use of side rails } \\
\text { - Night light in Room } \\
\text { - Consider change of bed to crib/other bed type as developmentally } \\
\text { appropriate. } \\
\text { - Offer child life consult as appropriate to provide diversional activity. } \\
\text { - Apll light within reach of caregiver/patient. } \\
\text { - Room free of clutter. } \\
\text { - Assess to need to move closer to nursing station for } \\
\text { unattended/high risk fall }\end{array}$ \\
\hline
\end{tabular}

Imagem 1: 2006 Razmus \& Wilson

The Humpty Dumpty Falls Scale (2007)- Diferencia a população pediátrica do hospital em categorias de baixo ou alto risco de quedas com base em fatores específicos. Sendo eles: idade, sexo, diagnóstico, deficiências cognitivas, fatores do paciente e ambientais (histórico de quedas, a colocação de cama adequada a idade da criança, equipamento / mobiliário e uso de dispositivos de assistência), resposta a cirurgias / sedação / anestesia e uso de medicação. 


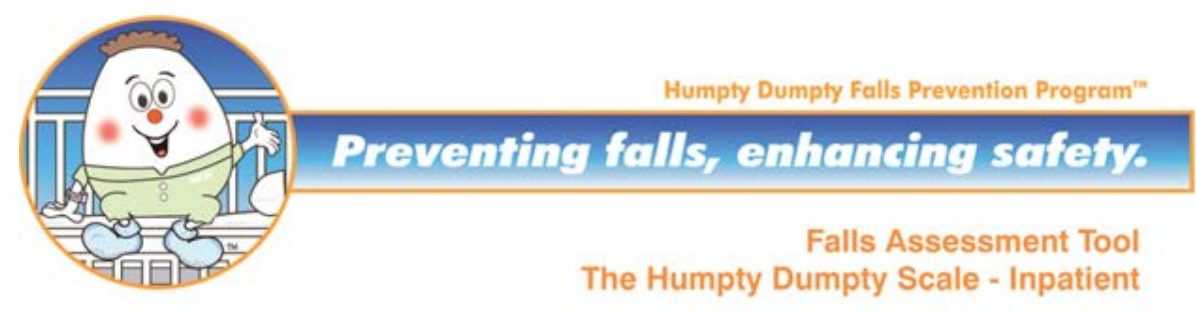

\begin{tabular}{|c|c|c|}
\hline Parameter & Criteria & Score (circle) \\
\hline \multirow[t]{4}{*}{ Age } & Less than 3 years old & 4 \\
\hline & 3 to less than 7 years old & 3 \\
\hline & 7 to less than 13 years old & 2 \\
\hline & 13 years and above & 1 \\
\hline \multirow[t]{2}{*}{ Gender } & Male & 2 \\
\hline & Female & 1 \\
\hline \multirow[t]{4}{*}{ Diagnosis } & Neurological Diagnosis & 4 \\
\hline & $\begin{array}{l}\text { Alterations in Oxygenation (Respira- } \\
\text { tory Diagnosis, Dehydration, Anemia, } \\
\text { Anorexia, Syncope/Dizziness, etc.) }\end{array}$ & 3 \\
\hline & Psych/Behavioral Disorders & 2 \\
\hline & Other Diagnosis & 1 \\
\hline \multirow{3}{*}{$\begin{array}{l}\text { Cognitive } \\
\text { Impairments }\end{array}$} & Not Aware of Limitations & 3 \\
\hline & Forgets Limitations & 2 \\
\hline & Oriented to own ability & 1 \\
\hline \multirow[t]{4}{*}{$\begin{array}{l}\text { Environmental } \\
\text { Factors }\end{array}$} & $\begin{array}{c}\text { History of Falls or } \\
\text { Infant-Toddler Placed in Bed }\end{array}$ & 4 \\
\hline & $\begin{array}{l}\text { Patient uses assistive devices or } \\
\text { Infant-Toddler in Crib or } \\
\text { Furniture/Lighting (Tripled room) }\end{array}$ & 3 \\
\hline & Patient Placed in Bed & 2 \\
\hline & Outpatient Area & 1 \\
\hline \multirow{3}{*}{$\begin{array}{l}\text { Response to } \\
\text { Surgery/Sedation/ } \\
\text { Anesthesia }\end{array}$} & Within 24 hours & 3 \\
\hline & Within 48 hours & 2 \\
\hline & More than 48 hours/None & 1 \\
\hline \multirow[t]{3}{*}{$\begin{array}{l}\text { Medication } \\
\text { Usage }\end{array}$} & $\begin{array}{c}\text { Multiple usage of: } \\
\text { Sedatives (excluding ICU patients } \\
\text { sedated and paralyzed) } \\
\text { Hypnotics } \\
\text { Barbiturates } \\
\text { Phenothiazines } \\
\text { Antidepressants } \\
\text { Laxatives/Diuretics } \\
\text { Narcotic } \\
\end{array}$ & 3 \\
\hline & One of the meds listed above & 2 \\
\hline & Other Medications/None & 1 \\
\hline
\end{tabular}

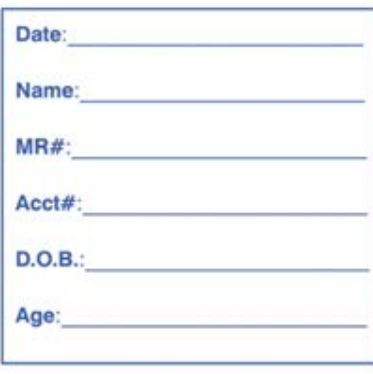

At risk for falls

If score is 12 or Above

Minimum Score 7 Maximum Score 23

ar Patient Falls Safety Protocol on back
YOUR LOGO HERE

Imagem 2: 2007 Rodriguez et AL.

Devemos ter atenção com as crianças com histórico de queda de baixa altura resultando em lesões múltiplas e/ou graves como fratura de crânio, edema cerebral e hematoma subdural, que são incompatíveis com a história. É evidenciado em diversos estudos que queda de baixas alturas não produz fraturas múltiplas e que as clinicamente significantes são incomuns, portanto nesses casos o histórico do paciente deve ser seriamente questionado, pois pode se suspeitar de abuso ou maus tratos infantil.

\section{Conclusão}

Prevenção de quedas na população pediátrica é difícil devido à imprevisibilidade das quedas como resultado de uma alteração cognitiva, crescimento e desenvolvimento da criança.

Evidencia-se a importancia da detecção dos pacientes de risco, da utilização de protocolos de prevenção de quedas e de realizarem-se adequações físicas do ambiente e do mobiliário hospitalar com vista a segurança do paciente durante a internação. 


\title{
Fall in Pediatrics: A challenge for the multidisciplinary team.
}

\begin{abstract}
:
Objective: The fall event is currently considered a major challenge to improve the quality of care in health so this study was motivated to generate data to support improving the quality of care in health.

Method: A survey of the relevant falling literature in pediatric settings. The articles were selected from Scielo data base, in Portuguese and English Results: Although falls are the leading cause of unintentional injuries in children, published works are scarce. The falls are clearly related to stage of development in children's, the level of injury is low and within the hospital most of the falls are due to the environmental conditions of health service. Conclusion: This study highlights the importance of the detection of patients at risk through validated tolls for the pediatric population, the use of fall prevention and change of organizational culture focused on patient safety protocols.
\end{abstract}

Keywords: fall, pediatrics, indicators.

\section{Referências}

1. HELFER E R, SLOVIS L T, BLACK M. Injuries resulting when small children fall out of bed. Pediatrics 1977;60;533.

2. HITCHO B E, KRAUSS J M, BIRGE S, DUNAGAN C W, FISCHER I, JOHNSON S, NAST A P, COSTANTINOU E, FRASER J V. Characteristics and circunstances of falls in a hospital setting. J Gen Intern Med 2004;19: 732-739.

3. LYONS J T, OATES K R. Falling out of bed: A relatively benign occurrence. Pediatrics 1993; 92; 125

4. MARIN F H, BOURIE P, SAFRAN C. Desenvolvimento de um sistema de alerta para prevenção de quedas em pacientes hospitalizados. Rev. Latino-am. Enfermagem. 2000;8(1):27-32.

5. MONSON A S, HENRY E, LAMBERT K D, SCHMUTZ N, CHRISTENSEN D R. Inhospital fall of newborn infants: data a multihospital health care system. Pediatrics 2008; 122;e277.

6. PAIVA S M C M, PAIVA R A S, BERTI W H, CAMPANA O A. Caracterização das quedas de pacientes segundo notificação em boletins de eventos adversos. Rev. Esc Enferm USP 2010; 44(1):134-8.

7. RAZMUS I, WILSON D, SMITH R, NEWMAN E. Falls in hospitalized children. Pediatric Nursing 2006; vol 32.

8. RODRIGUEZ H D, MESSMER R P, WILLIAMS D P, ZELLER A R, WILLIAMS R A, WOOD M, HENRY M. The Humpty Dumpty falls sacale: a case-control study. JSPN 2009; 14(1):23- 32

9. VIEIRA M P A, KURCGANT P. Indicadores de qualidade no gerenciamento de recursos humanos em enfermagem: elementos constitutivos segundo percepção de enfermeiros. Acta Paul Enferm 2010; 23(1):11-5

10. WARDA L. Pediatric injuries in the hospital setting. Paediatr Child Health 2004:9(5)331-334.

11. LYONS J T, OATES K R. Falling out of bed: A relatively benign occurrence. Pediatrics 1993; 92; 125 